\title{
Razonamiento mecánico, memoria de trabajo y velocidad de procesamiento
}

\author{
Mechanical reasoning, working memory and processing speed
}

\author{
Irene Injoque-Ricle ${ }^{\mathrm{a}, \mathrm{b}, *}$, Jésica Formoso ${ }^{\mathrm{a}, \mathrm{b}}$, Alejandra Calero ${ }^{\mathrm{a}, \mathrm{b}}$, Guido Caruso ${ }^{\mathrm{a}}$, \\ Andrea Álvarez Drexler ${ }^{\mathrm{a}}$, Juan Pablo Barreyro ${ }^{\mathrm{a}, \mathrm{b}}$ \\ aDepartamento de Procesos Básicos, Instituto de Investigaciones, Facultad de Psicología, \\ Universidad de Buenos Aires, Argentina \\ bConsejo Nacional de Investigaciones Científicas y Técnicas (CONICET), Argentina
}

\section{Resumen}

Antecedentes: el razonamiento mecánico (RM) es la habilidad para identificar los componentes de un sistema y comprender como estos interactúan para que el sistema funcione. Distintos sistemas mecánicos, como de rueda y de engranaje, requieren diferentes tipos de razonamiento. Objetivo: este trabajo busca analizar la relación de la MT y la velocidad de procesamiento (VP) con el RM. Método: para ello, se administraron a 173 estudiantes universitarios 30 problemas de RM del test DAT, el sub-test Búsqueda de Símbolos de la batería WAIS-III para evaluar VP y las baterías BIMET-V y BIMET-VE para evaluar los componentes verbal y visoespacial de la MT. Resultados: los análisis de regresión mostraron que las variables propuestas no predicen el rendimiento en problemas de engranajes, mientras que la MT viso-espacial y la VP predicen el rendimiento en problemas de rueda. Discusión: los resultados sugieren que los problemas de engranaje tienen una baja demanda ejecutiva al poder resolverse de forma fraccionada, mientras que los problemas de rueda requieren sostener en memoria la totalidad de los componentes que forman parte del sistema, a la vez que requieren mayor carga atencional.

Palabras clave: razonamiento mecánico, memoria de trabajo, memoria de corto plazo, velocidad de procesamiento.

Para citar este artículo:

Injoque-Ricle, I., Formoso, J., Calero, A., \& Caruso, G. (2019). Razonamiento mecánico, memoria de trabajo y velocidad de procesamiento. Liberabit, 25(1), 71-84. doi: https://doi.org/10.24265/ liberabit.2019.v25n1.06

\section{Abstract}

Background: mechanical reasoning (MR) is the ability to identify the components of a system and understand how they interact in order for the system to work. Different mechanical systems, such as those of wheels and gears, require different types of reasoning. Objective: this study seeks to analyze the relationship that working memory (WM) and processing speed (PS) have with MR. Method: one hundred seventy-three (173) university students were assessed through the DAT test which consisted of $30 \mathrm{MR}$ problems, the symbol search subtest of the WAIS-III battery for assessing the PS, and the BIMET-V and BIMET-VE batteries to evaluate the WM verbal and visuospatial components. Results: regression analyses showed that the proposed variables do not predict the performance in gear problems, while the visuospatial WM and the PS predict the performance in wheel problems. Discussion: the results suggest that gear problems have a low executive demand as they can be solved in a fractional way, while wheel problems require to hold in memory all the components that are part of the system and, at the same time, require a higher attentional load.

Keywords: mechanical reasoning, working memory, short-term memory, processing speed.

Este es un artículo Open Access bajo la licencia Creative Commons Atribución-NoComercial-CompartirIgual 4.0

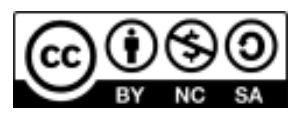




\section{Introducción}

El razonamiento mecánico es un proceso psicológico de orden superior a través del cual una persona puede identificar los componentes principales de un sistema mecánico y su relevancia para el funcionamiento del mismo e inferir cómo interactúan entre ellos. Esta habilidad forma parte de la inteligencia espacial (Hegarty, 2010; Hegarty \& Tarampi, 2015) definida como un pensamiento espacial adaptativo que se ocupa de cómo las personas adquieren, procesan y utilizan el conocimiento espacial, y se aplica a actividades como inferir el funcionamiento de sistemas a partir de la lectura de descripciones del funcionamiento de maquinarias 0 diagramas estáticos del mismo, operar con maquinaria compleja, así como diagnosticar fallas y diseñar nuevas maquinarias (Rouse \& Morris, 1986; Williams, Hollan, \& Stevens, 1983).

Quienes se destacan en razonamiento mecánico tienen facilidad para aprender y comprender los principios de funcionamiento y separación de dispositivos complejos, una habilidad que es frecuentemente implementada en áreas como la ingeniería y es evaluada en los tests de aptitudes, como el Test de Aptitudes Diferenciales (Bennett, Seashore, \& Wesman, 1992) o la Batería para la Evaluación de Aptitudes (Arribas, Santamaría, Sánchez-Sánchez, \& Fernández-Pinto, 2013). Numerosos estudios (Andersen, 2014; Wai \& Kell, 2017; Ackerman, 2014; Stieff \& Uttal, 2015) encontraron que las habilidades espaciales predicen el éxito en las disciplinas académicas de Ciencia, Tecnología, Ingeniería y Matemáticas (CTIM, STEM en inglés), las cuales se encuentran en la base del proyecto de educación STEM en Estados Unidos, en tanto se las considera el medio para el diseño y creación de objetos y sistemas para la resolución de problemas económicos y tecnológicos (Kelley \& Knowles, 2016; English, 2016).

Liu y Schunn (2017) muestran que niveles altos de razonamiento mecánico se asocian con el uso frecuente y complejo de estrategias matemáticas, por lo que sugieren que esta habilidad puede ser el camino a través del cual se puede mejorar el uso adaptativo de estrategias matemáticas. Por estas razones, es importante conocer los procesos cognitivos subyacentes que inciden en la habilidad de razonar a partir de sistemas mecánicos.

En este sentido, la memoria de trabajo y la velocidad de procesamiento se consideran variables cognitivas que impactan de manera directa en los procesos cognitivos de orden superior como el razonamiento y la comprensión. Existen distintos sistemas mecánicos, cada uno de los cuales hace uso de diferentes tipos de razonamiento en términos de su dependencia de estrategias y habilidades espaciales específicas. Uno de ellos es el sistema de engranajes, que requiere de la habilidad de inferir el movimiento de una pieza de un engranaje a partir del movimiento de otra pieza adyacente. Estos problemas pueden ser resueltos utilizando una estrategia de pasos sucesivos, que implica una cadena de inferencias de los movimientos de cada engranaje por separado (Hegarty \& Sims, 1994).

De acuerdo con Hegarty y Kozhevnikov (1999) el tipo de razonamiento necesario para interpretar estos sistemas o inferir el movimiento de una o más piezas, tiene una baja demanda ejecutiva de memoria de trabajo. Esto se debe a que los problemas no se representan globalmente, sino de manera fraccionada, pudiendo inferirse el funcionamiento global analizando una parte tras otra. En la Figura 1a, se muestra un ejemplo de un problema de este tipo. Otro tipo de sistema son los de rueda, que requieren la habilidad de determinar el movimiento del conjunto a partir de la combinación de los movimientos individuales de las partes que lo componen. En este caso, varios componentes limitan la acción de otros, por lo que el movimiento de todo el sistema debe ser visualizado de manera conjunta haciendo que no sea posible representar mentalmente el problema de forma fragmentada. Para Hegarty y Kozhevnikov (1999), la capacidad de almacenamiento y procesamiento 

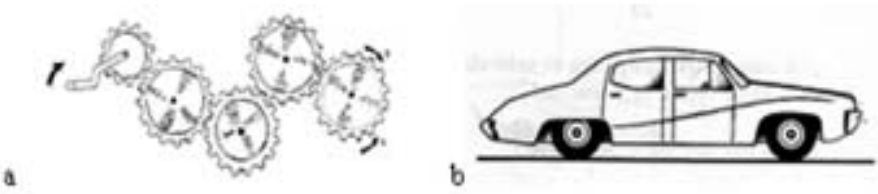

Figura 1. a) Problema de engranaje: se pide que diga en qué dirección gira el último engranaje de la cadena (extraído de Hegarty, \& Sims, 1994). b) Problema de ruedas: se pide que indique si el peso sobre las ruedas delanteras es mayor, menor o igual que el peso sobre las traseras cuando el automóvil frena (extraído de Bennet et al., 1992).

concurrente de memoria de trabajo espacial determina el éxito en la resolución de este tipo de problemas. En la Figura 1b se muestra un ejemplo de un problema de rueda.

La memoria de trabajo es un sistema de almacenamiento y procesamiento concurrente al servicio de tareas cognitivas complejas, tales como el razonamiento y la comprensión (Baddeley, 2010, 2018). Según el modelo clásico de Baddeley y Hitch (1974; Baddeley, 2018), está formada por tres componentes: (1) el ejecutivo central y dos subsistemas esclavos, (2) el bucle fonológico y (3) la agenda visoespacial. El ejecutivo central es un sistema de atención y control que monitorea y regula el procesamiento de información en los dos subsistemas. Se encarga de activar los procesos necesarios para manipular la información almacenada en los subsistemas esclavos -verbal en el caso del bucle fonológico y visoespacial en el caso de la agenda visoespacial (Baddeley, 2010, 2018; Baddeley \& Hitch, 1974). El bucle fonológico almacena por breves períodos de tiempo una cantidad limitada de información verbal, y la agenda visoespacial realiza la misma acción con información visual y espacial (Baddeley, 2010, 2018; Baddeley \& Hitch, 1974). El sostenimiento de información en los subsistemas sin manipulación activa por parte del sistema ejecutivo, refiere al concepto tradicional de memoria de corto plazo verbal y visoespacial, respectivamente.

Por su parte, la velocidad de procesamiento refiere a la rapidez con que un sujeto ejecuta una tarea cognitiva simple y relativamente automatizada, como puede ser una simple prueba de tiempos de reacción (Jensen, 2006; Sheppard \& Vernon, 2008). La misma aumenta durante la infancia (Formoso, Jacubovich, Injoque-Ricle, \& Barreyro, 2018; Fry \& Hale, 2000; Mella, Fagot, Lecerf, \& Ribaupierre, 2015) y tiende a disminuir con la edad en sujetos adultos (An et al., 2018). La velocidad de procesamiento incide sobre la capacidad de un sujeto para codificar, transformar y recuperar información (Conway, Cowan, Bunting, Therriault, \& Minkoff, 2002), por lo que ha sido vinculada a la capacidad de procesamiento concurrente de la memoria de trabajo (Demetriou et al., 2014), y ha sido asociada fuertemente con medidas de inteligencia general (Engelhardt et al., 2016). Sin embargo, no se han encontrado publicaciones que evalúen el rol de la velocidad de procesamiento en el razonamiento mecánico.

Por otro lado, aunque se ha estudiado la relación del razonamiento mecánico con la memoria de trabajo, son pocas las investigaciones que han abordado esta temática (Hegarty, 2004; Hegarty \& Kozhevnikov, 1999; Hegarty \& Steinhoff, 1997). Hegarty y Steinhoff (1997) evaluaron el rendimiento de sujetos con baja y alta capacidad espacial en tareas de verificación del movimiento (polea y palanca, y engranajes). Este tipo de tareas evalúan el razonamiento mecánico de los participantes al pedir que definan si ciertas afirmaciones acerca del movimiento son correctas o incorrectas. A la mitad de los participantes de ambos grupos (baja y alta capacidad) se les permitió realizar notas al margen de las láminas como ayuda para la realización de la tarea. 
Los resultados obtenidos mostraron, en primer lugar, que existen diferencias entre los grupos en cuanto al rendimiento a favor de los sujetos con mayor capacidad espacial. Adicionalmente, los individuos con baja capacidad muestran diferencias en el desempeño a favor de los participantes a los que se les permitió tomar notas, mientras que los sujetos con alta capacidad espacial no mostraron diferencias entre uno y otro grupo. Los autores concluyen que el menor rendimiento en las tareas de procesamiento mecánico de los sujetos con baja capacidad espacial puede deberse a limitaciones en la memoria de trabajo visoespacial, y que tomar notas funciona como un apoyo externo al sistema de memoria, mientras que esto no resultaría necesario para los participantes de alta capacidad. Adicionalmente, en los sujetos con baja capacidad visual se observó una asociación entre la proporción de errores, así como los tiempos de reacción y la distancia entre los componentes del esquema de poleas o engranajes (Hegarty \& Sims, 1994). Este patrón no se observó en los participantes con mayor capacidad visual.

En estudios subsiguientes (Hegarty, 2004; Hegarty \& Steinhoff, 1997), se utilizó el paradigma de la doble tarea para medir la interferencia sobre el razonamiento mecánico de mantener información visoespacial activa en la memoria de trabajo, específicamente en la agenda visoespacial. A su vez, compararon el efecto de utilizar información visoespacial respecto de utilizar información verbal como interferencia, involucrando así al bucle fonológico. Los autores sugirieron que el razonamiento mecánico depende de representaciones almacenadas temporariamente y procesadas en la agenda visoespacial, excluyendo al bucle fonológico.

El objetivo de este trabajo es estudiar la relación de la memoria de trabajo y la velocidad de procesamiento con el razonamiento mecánico. Particularmente, indagar si distintos componentes de la memoria de trabajo tienen el mismo peso en la resolución de problemas de razonamiento mecánico específicos.

\section{Método}

\section{Participantes}

Se trabajó con 173 estudiantes universitarios de entre 18 y 35 años del primer año de la carrera de Psicología (Media $=21.44 ; D E=2.95$ ) que participaron de manera voluntaria y anónima ante el estudio, luego de que se les explicaran los objetivos y características del mismo. Se utilizó un muestreo de tipo no probabilístico por conveniencia. Un 54.30\% de los participantes eran mujeres ( $n=94$ ) y un $45.70 \%$ varones. Los estudiantes fueron contactados a través de los profesores, quienes conocían los objetivos y características de la investigación y dieron su consentimiento para que la participación en el estudio fuese equivalente a la realización de un trabajo práctico de la materia en el caso que decidiesen participar.

\section{Materiales}

Tarea de Razonamiento Mecánico (Bennett et al., 1992). Se administraron 30 problemas de la prueba Razonamiento Mecánico del Test de Aptitudes Diferenciales. Se incluyeron 15 problemas que requieren la habilidad de inferir la fuerza necesaria para mover un objeto con determinado peso a partir del uso de un engranaje y 15 problemas que requieren la habilidad de determinar el movimiento de un sistema a partir de la interacción de los movimientos de las partes que lo componen. Todos los problemas son presentados visualmente en una imagen tridimensional y se dan tres opciones de respuesta, esto permite obtener una puntuación de razonamiento mecánico general, así como de razonamiento mecánico de engranaje y de razonamiento mecánico de rueda.

Batería Informatizada de Memoria de Trabajo (Barreyro, Injoque-Ricle, Formoso, \& Burin, 2019; Injoque-Ricle, Barreyro, Formoso, \& Burin, 2018). Permite realizar una medición exhaustiva de los componentes de la memoria de trabajo, brindando puntuaciones de memoria corto plazo verbal, memoria de trabajo verbal o de alta demanda ejecutiva, memoria 
de corto plazo visoespacial y memoria de trabajo visoespacial o de alta demanda ejecutiva, ya que la resolución de las tareas de memoria de trabajo verbal y visoespacial no solo requieren del sostenimiento de la información en los respectivos almacenes, sino de la manipulación de esa información mediada por el ejecutivo central.

La batería se encuentra formada por la Batería Informatizada de Memoria de Trabajo verbal (BIMeTv; Barreyro et al., 2019) y por la Batería Informatizada de Memoria de Trabajo visoespacial (BIMeT-ve; Injoque-Ricle et al., 2018). Todas las pruebas cuentan con niveles de dificultad crecientes y se interrumpen en función del desempeño del sujeto: cuando realiza de manera incorrecta al menos dos de los tres ensayos de un nivel.

La BIMeT-v está compuesta por 1) Amplitud de dígitos $(\alpha=.81)$, la cual tiene como objetivo evaluar la capacidad de almacenamiento de información verbal en el bucle fonológico, es decir, la memoria de corto plazo verbal. Se presentan visualmente en una pantalla de computadora, uno por vez, números del 1 al 9, los números se presentan por $2000 \mathrm{~m}$ y el intervalo entre ellos es de un segundo. El sujeto tiene que retener dichos números y cuando aparezca la palabra «recuerdo» tiene que indicar en una matriz de números cuáles le fueron presentados, en el exacto orden en el que aparecieron. En la Figura 2a se presenta un ejemplo del nivel 3. 2) Amplitud de letras $(\alpha=.83)$, el cual tiene el mismo objetivo $\mathrm{y}$ procedimiento que Amplitud de dígitos, salvo que, en lugar de números, los estímulos son letras. En la Figura 2b se presenta un ejemplo del nivel 3. 3) Amplitud rápida $(\alpha=.78)$, el cual tiene como objetivo medir la capacidad de almacenamiento y procesamiento concurrente de información verbal manipulada por el ejecutivo central y almacenada temporalmente por el bucle fonológico, brindando una medida de memoria de trabajo verbal. Se le indica al sujeto que se le van a presentar una serie de letras no se le indican cuántas- y que tiene que recordar las últimas letras de la serie -en el caso del nivel 2, las últimas dos letras-, cada letra aparece en el centro de la pantalla por un segundo y el intervalo de tiempo entre estímulos es de $500 \mathrm{~m}$. Cuando aparece la palabra «recuerdo» el sujeto tiene que indicar en una matriz de letras cuáles son las últimas dos letras presentadas -en el caso del nivel 2- en el exacto orden en el que aparecieron. En la Figura 2c se presenta un ejemplo del nivel 3.4) Ordenamiento letra-dígito ( $\alpha=.84$ ), el cual tiene el mismo objetivo que Amplitud rápida. En este caso se le presenta en la pantalla de la computadora números y letras mezclados y cuando aparece la palabra «recuerdo» el sujeto tiene que indicar en una matriz de números y letras, primero las letras que le fueron presentadas en orden alfabético, y luego los números en orden ascendente. Cada estímulo se presenta por $2000 \mathrm{~ms}$, y el intervalo entre ellos es de un segundo. En la Figura 2d se presenta un ejemplo del nivel 3.

La BIMeT-ve está formada por cuatro pruebas. 1) Bloques simple $(\alpha=.81)$ es una versión de la tarea Bloques de Corsi (1972), la cual mide la capacidad de almacenamiento de información en la agenda visoespacial, es decir, de memoria de corto plazo visoespacial. Se presentan en una pantalla de computadora nueve bloques blancos distribuidos de manera no uniforme. En cada ensayo, y de uno en uno, los cubos se vuelven rojos creando una secuencia. El sujeto tiene que reproducir dicha secuencia haciendo click con el mouse sobre los bloques que cambiaron de color, en el orden exacto en el que fueron presentados. En el primer nivel cambia un solo bloque de color; en el segundo nivel, la secuencia es de dos ítems a recordar y aumenta de uno en uno hasta el noveno nivel, en el que la secuencia a recordar es de nueve bloques. Cada bloque de color aparece por 1000 m. En la Figura 3a se muestra un ejemplo de un ensayo del nivel 4. 2) Estrella simple $(\alpha=.71)$, la cual se trata de una medida adicional de memoria de corto plazo visoespacial. Consiste en la presentación en una pantalla de computadora de una estrella azul de siete puntas. En una de las puntas, y de a uno por vez, aparece un círculo rojo. El sujeto tiene que recordar 
en qué punta de la estrella apareció cada punto rojo y el orden exacto de presentación, indicando el lugar al hacer click con el mouse. Cada círculo aparece por 1000 m. En la Figura 3b se muestra un ejemplo de un ensayo del nivel 4. 3) Bloques con interferencia $(\alpha=.75)$, la cual es la versión compleja de Bloques simples. Permite medir la capacidad de almacenamiento y procesamiento concurrente de información almacenada en la agenda visoespacial por medio de la acción del ejecutivo central, brindando una medida de memoria de trabajo visoespacial. El procedimiento difiere en que entre la finalización de la presentación de la secuencia de bloques y el recuerdo se presenta una tarea secundaria que dura $2000 \mathrm{~m}$. Esta tarea consiste en identificar entre tres polígonos, cuál es el más complejo -el que más lados tiene- haciendo click con el mouse sobre uno de ellos. En la Figura 3c se presenta un ejemplo del nivel 3. 4) Estrella con interferencia $(\alpha=.82)$, siendo la versión compleja de Estrella simple. Evalúa la memoria de trabajo visoespacial. La tarea secundaria consiste en la presentación de dos relojes de aguja con horas diferentes y un reloj digital que marca la misma hora que uno de los relojes de aguja. Los relojes aparecen en pantalla por $2000 \mathrm{~m}$ y el sujeto tiene que identificar cuál de los dos relojes de aguja marca la misma hora que el reloj digital, y hacer click con el mouse sobre este. En la Figura 3d se presenta un ejemplo del nivel 3.
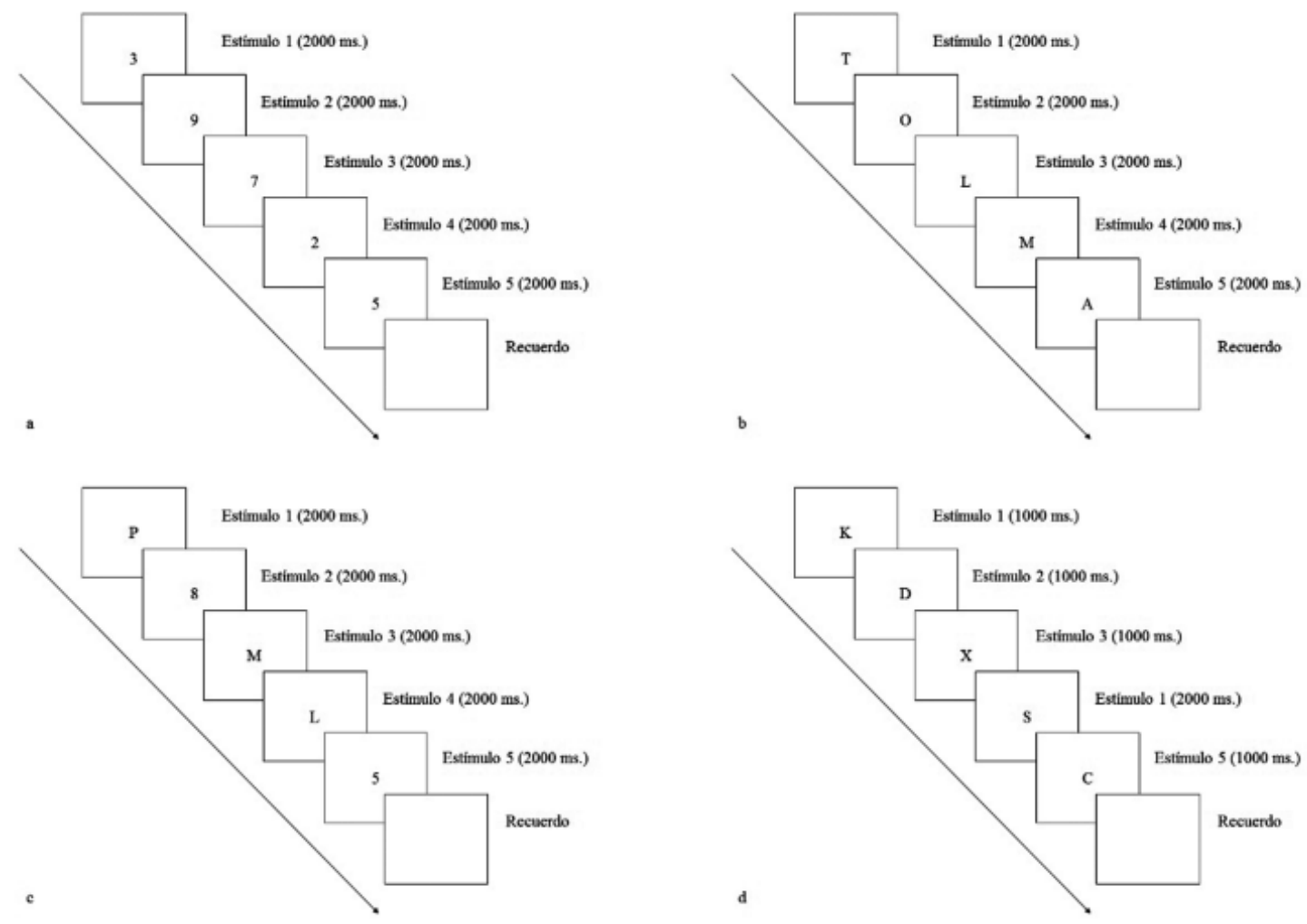

Figura 2. Ejemplos del nivel 3 de las tareas de la BIMeT-v. a) Amplitud de dígitos, b) Amplitud de letras, c) Amplitud rápida y d) Ordenamiento letra-dígitos. 

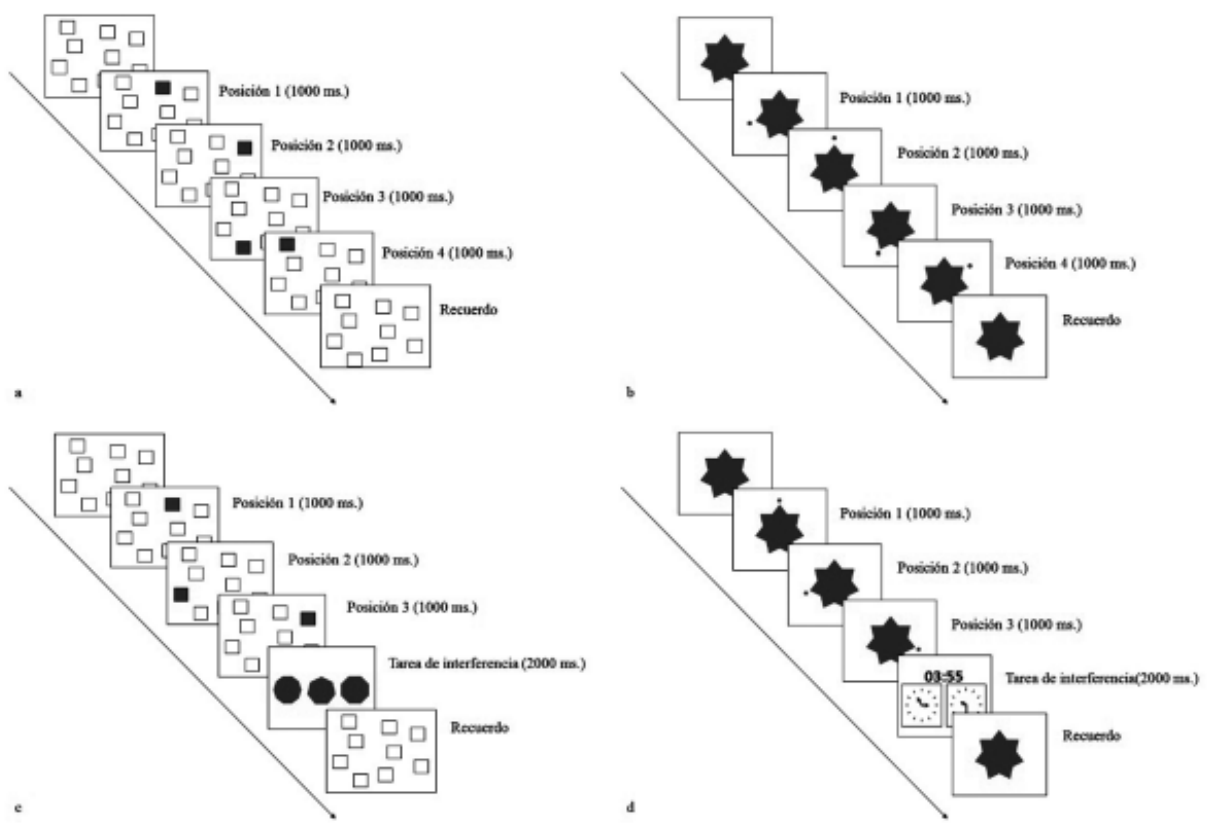

Figura 3. Ejemplos del nivel 3 de las tareas de la BIMeT-ve.
a) Bloques simples, b) Estrella simple, c) Bloques con interferencia y d) Estrella con interferencia.

Búsqueda de símbolos (Wechsler, 2003). Forma parte de la Escala de Inteligencia para Adultos de Wechsler. Permite medir la velocidad, fluencia y precisión con la que un sujeto responde a una tarea. Se presentan problemas con dos grupos de símbolos: un grupo objetivo y un grupo de búsqueda. El sujeto tiene que indicar si alguno de los símbolos del grupo objetivo se encuentra en el grupo de búsqueda en un tiempo determinado. Se toma como una medida de velocidad de procesamiento.

\section{Procedimiento}

Se trata de un estudio descriptivo, de corte transversal, con un diseño intrasujeto, donde a cada participante se le administró cada prueba individualmente en una única sesión de aproximadamente 40 minutos. La evaluación fue realizada por evaluadores capacitados en un ambiente libre de ruidos y distracciones, en el Instituto de Investigaciones de la Facultad de Psicología. Se contrabalanceó la presentación de los instrumentos para contrarrestar el efecto que la fatiga puede tener sobre el desempeño de los sujetos.

\section{Análisis de datos}

En primer lugar, se realizaron análisis de correlaciones utilizando el estadístico $r$ de Pearson para determinar el grado de asociación de las variables de memoria de trabajo y velocidad de procesamiento con las medidas de razonamiento mecánico. En segundo lugar, se realizaron análisis de regresión lineal múltiples para establecer el poder predictivo de los componentes de la memoria de trabajo y la velocidad de procesamiento sobre el razonamiento mecánico global y los dos subtipos incluidos en este estudio.

\section{Resultados}

En la Tabla 1 se exponen los estadísticos descriptivos de las variables incluidas en el estudio. La puntuación de razonamiento mecánico se obtuvo de la suma de los puntajes de cada subprueba de engranaje y de rueda, según el procedimiento de 
Bennet et al. (1992). Por otro lado, se estimaron las puntuaciones de memoria de corto plazo verbal y visoespacial, así como de memoria de trabajo verbal y visoespacial promediando las dos pruebas que miden el mismo constructo, siguiendo el procedimiento de Barreyro et al. (2019) e Injoque-Ricle et al. (2018).

Tabla 1

Estadísticos descriptivos de las variables en estudio

\begin{tabular}{lcccc}
\hline & Media & $D E$ & Asimetría & Curtosis \\
\hline RM & 8.91 & 2.65 & .01 & -.09 \\
RM engranaje & 5.32 & 1.59 & -.04 & -.07 \\
RM rueda & 3.60 & 1.61 & .01 & -.50 \\
MCP verbal & 13.65 & 3.05 & -.32 & .24 \\
MT verbal & 8.05 & 2.30 & .27 & .12 \\
MCP visoespacial & 8.15 & 2.59 & -.11 & -.60 \\
MT visoespacial & 3.48 & 1.90 & .64 & -.07 \\
Velocidad de procesamiento & 31.46 & 8.45 & -.56 & 2.51 \\
\hline
\end{tabular}

Nota: $\mathrm{RM}=$ Razonamiento mecánico; $\mathrm{MCP}=$ Memoria de corto plazo; $\mathrm{MT}=$ Memoria de trabajo.

El análisis de correlaciones mostró asociaciones positivas significativas entre el razonamiento mecánico y las variables restantes (ver Tabla 2). Lo mismo se observó al analizar la asociación entre las variables y el razonamiento mecánico de engranaje (ver Tabla 2).
Por su parte, en el caso del razonamiento mecánico de rueda solo se encontraron asociaciones positivas significativas con memoria de trabajo visoespacial $(r=.24, p<.01)$ y con velocidad de procesamiento $(r=.22, p=<.01)$ (ver Tabla 2).

Tabla 2

Correlaciones entre las puntuaciones de razonamiento mecánico con las de memoria de trabajo y velocidad de procesamiento

\begin{tabular}{llcc}
\hline & RM & RM engranaje & RM rueda \\
\hline Memoria de corto plazo verbal & $.18^{*}$ & $.24^{* *}$ & .07 \\
Memoria de trabajo verbal & $.24^{* *}$ & $.25^{* *}$ & .14 \\
Memoria de corto plazo visoespacial & $.25^{* *}$ & $.28^{* *}$ & .13 \\
Memoria de trabajo visoespacial & $.30^{* *}$ & $.26^{* *}$ & $.24^{* *}$ \\
Velocidad de Procesamiento & $.23^{* *}$ & $.16^{*}$ & $.22^{* *}$ \\
\hline
\end{tabular}

Nota: $\mathrm{RM}=$ Razonamiento mecánico; $* p<.05 ; * * p<.01$ 
Dado que se observaron patrones de correlación diferentes con cada subtipo de razonamiento mecánico, se realizaron análisis de regresión donde se pusieron a prueba dos modelos para estudiar el poder predictivo de los componentes de la memoria de trabajo y la velocidad de procesamiento sobre el razonamiento mecánico de engranaje y de rueda por separado. En el primer modelo se estudió el poder predictivo de los cuatro componentes de la memoria de trabajo y la velocidad de procesamiento sobre el razonamiento mecánico de engranaje. Si bien el mismo mostró ser significativo $\left(\mathrm{F}_{(5)}=4.73, p<.01, \mathrm{R}_{\mathrm{c}}^{2}=.12\right)$, al analizar los efectos de cada una de las variables predictoras, se observa que ninguna de estas tiene un efecto significativo sobre las puntuaciones del razonamiento mecánico de engranaje. El segundo modelo, que busca determinar el poder predictivo de las variables en estudio sobre el razonamiento mecánico de rueda, también resultó significativo $\left(\mathrm{F}_{(5)} 3.80, p<.01, \mathrm{R}^{2}{ }_{\mathrm{c}}=\right.$ .10). En este caso, al analizar los efectos de cada una de las variables predictoras, se observa que las únicas que predicen significativamente el comportamiento de razonamiento mecánico de rueda son memoria de trabajo visoespacial $(\beta=.22)$ y velocidad de procesamiento $(\beta=.22)$ (ver Tabla 3$)$.

Tabla 3

Modelos de regresión lineal múltiple

\begin{tabular}{llcccccccc}
\hline & $\beta$ & $t$ & $p$ & $F$ & $M S E$ & $g l$ & $p$ & $\mathrm{R}_{\mathrm{c}}^{2}$ \\
\hline Modelo 1 & Ajuste & & & & 4.73 & 2.28 & 5 & .00 & .12 \\
& MCP verbal & .04 & .40 & .69 & & & & & \\
& MT verbal & .14 & 1.48 & .14 & & & & & \\
& MCP visoespacial & .14 & 1.50 & .14 & & & & & \\
& MT visoespacial & .12 & 1.43 & .15 & & & & & \\
& VP & .05 & .59 & .55 & & & & & \\
\cline { 2 - 7 } Modelo 2 & Ajuste & & & & 3.80 & 2.41 & 5 & .00 & \\
& MCP verbal & -.07 & -.75 & .45 & & & & & \\
& MT verbal & .04 & .45 & .65 & & & & & \\
& MCP visoespacial & -.02 & -.24 & .81 & & & & & \\
& MT visoespacial & .22 & 2.48 & .01 & & & & & \\
& VP & .22 & 2.62 & .01 & & & & & \\
& & & & & & & & & \\
\end{tabular}

Nota: Variables dependientes: Modelo 1 = Razonamiento mecánico de engranaje; Modelo 2 = Razonamiento mecánico de rueda; MCP = Memoria de corto plazo; $\mathrm{MT}=$ Memoria de trabajo; VP = Velocidad de procesamiento.

\section{Discusión}

En la última década, la enseñanza de las disciplinas académicas CTIM (Ciencia, Tecnología, Ingeniería y Matemáticas) se ha vuelto de central importancia, especialmente siendo que se las considera asociadas al bienestar económico y el desarrollo tecnológico de los países (CITA). Estas, en particular Tecnología e Ingeniería, dependen en gran medida de la capacidad de los sujetos para razonar acerca del funcionamiento de sistemas mecánicos. Es por ello que identificar los procesos cognitivos que subyacen a esta habilidad adquiere una nueva relevancia. Tanto la memoria de trabajo como la velocidad de procesamiento suelen encontrarse en la base de numerosos procesos psicológicos de orden superior; sin embargo, son pocos los estudios que buscan analizar la relación entre 
estos y el razonamiento mecánico, entendido como representaciones espaciales internas de sistemas mecánicos que involucra el procesamiento analógico de imágenes, y puede disociarse del razonamiento basado en representaciones descriptivas o conocimiento explícito. Permite construir, mantener y transformar con precisión representaciones espaciales (Hegarty, 2004). Según Hegarty y Sims (1994) y Hegarty y Steinhoff (1997), resolver un problema mecánico se encuentra fuertemente relacionado con habilidades espaciales, como ser la imaginería visoespacial, pero no con habilidades verbales y plantea además que la imaginería visoespacial o animación mental está condicionada por la capacidad limitada de la memoria de trabajo, ya que los sujetos no pueden representar de manera simultánea el comportamiento de todos los componentes de un sistema mecánico (Hegarty, 2004). Hegarty y Steinhoff (1997) concluyeron que la baja capacidad de memoria de trabajo visoespacial afecta el rendimiento en tareas de razonamiento mecánico de polea y palanca, los cuales requieren la habilidad de inferir la fuerza necesaria para mover un objeto con determinado peso a partir del uso de una polea o una palanca. Por su parte, Hegarty y Kozhevnikov (1999) arribaron a la misma conclusión con tareas de razonamiento mecánico de engranaje, pero las inferencias se basaron en dividir a los sujetos con alta y baja capacidad de razonamiento mecánico y luego, a la mitad de ellos, permitirles tomar notas mientras que al resto no. Dicho estudio no incluye ninguna prueba de memoria de trabajo visoespacial que permita corroborar dicha conclusión. En estudios subsiguientes (Hegarty, 2004), se utilizó el paradigma de la doble tarea, tanto con información visoespacial como verbal, para indagar la interferencia que la retención de ambos tipos de información podía tener sobre el razonamiento mecánico, concluyendo que durante este proceso cognitivo superior la información es almacenada y procesada solo por la memoria de trabajo visoespacial.

A pesar de que los resultados alcanzados en estudios precedentes permiten inferir que la memoria de corto plazo visoespacial tiene incidencia en la resolución de tareas de razonamiento mecánico a diferencia de la memoria de corto plazo verbal, se carece de investigaciones que prueben esta relación de forma directa. Asimismo, tampoco se encuentran estudios que incluyan una variable íntimamente relacionada con la memoria de trabajo como lo es la velocidad de procesamiento, considerada una variable central en la resolución de todo tipo de tareas cognitivas complejas, que no se limita a la rapidez con la que se resuelven tareas simples, sino que es fundamental para la resolución, transformación y recuperación de información (Conway et al., 2002; Jensen, 2006; Sheppard \& Vernon, 2008).

Es por ello que el objetivo de este estudio fue indagar acerca del rol de los distintos componentes de la memoria de trabajo, incluso los verbales, y de la velocidad de procesamiento en el razonamiento mecánico y examinar si, según el tipo de razonamiento mecánico, dichas variables juegan un rol más preponderante que otras, tal como plantean los autores (Hegarty, 2004; Hegarty \& Kozhevnikov, 1999; Hegarty \& Sims, 1994; Hegarty \& Steinhoff, 1997).

Si bien los resultados mostraron asociaciones significativas entre el razonamiento mecánico y el resto de las variables en estudio que permitiría sostener la idea de que la velocidad de procesamiento de la información y los componentes de la memoria de trabajo (tanto verbales como visoespaciales, de almacenamiento y también de almacenamiento y procesamiento concurrente) están, al menos, asociados a los mecanismos generales del razonamiento mecánico y con la capacidad de razonar a partir de engranajes. Los mismos resultados no se encuentran al observar las correlaciones sobre la capacidad de razonar a partir de mecanismos de rueda, en donde se observa una asociación significativa con la velocidad de procesamiento y la memoria de trabajo visoespacial, estrictamente la capacidad de almacenamiento y procesamiento concurrente de información visual y espacial. Este resultado guarda cierta sintonía en cuanto al mecanismo de rueda y su relación con la memoria 
de trabajo visoespacial con aquellos presentados por Hegarty y Kozhevnikov (1999).

En cuanto a los modelos puestos a prueba a través de los análisis de regresión, permitieron indagar el poder predictivo de la velocidad de procesamiento y cada uno de los componentes de la memoria de trabajo. En primer lugar, al analizar los resultados del razonamiento a partir de mecanismos de engranaje no se encontraron efectos significativos. En cuanto a la memoria de trabajo, esto coincide con lo que sugieren Hegarty y Kozhevnikov (1999), quienes argumentan que los problemas de razonamiento mecánico de engranaje tienen una baja demanda ejecutiva de memoria de trabajo, ya que se pueden resolver de forma fraccionada. En cuanto a la velocidad de procesamiento, puede argumentarse que se trata de problemas más sencillos y que este proceso cognitivo no tiene una incidencia fuerte en su resolución.

Por su parte, los hallazgos en cuanto a la incidencia de los componentes de memoria de trabajo sobre la resolución de problemas que exigen razonar a partir de mecanismos de rueda, también coinciden con los planteos de Hegarty y Kozhevnikov (1999). Los resultados mostraron que solo la memoria de trabajo visoespacial predice el rendimiento en este tipo de tareas, ni la memoria de trabajo verbal ni ambos tipos de memoria a corto plazo. Los autores argumentan que a diferencia de los problemas de razonamiento mecánico de engranaje; en los de rueda, los sujetos necesitan representarse globalmente el problema incluyendo de forma simultánea todos los componentes del sistema, de forma que requieren de recursos de almacenamiento y procesamiento concurrente de la memoria de trabajo visoespacial. Asimismo, al tratarse de problemas más complejos en cuanto a su resolución, la demanda de velocidad de procesamiento es mayor, puesto que se requiere mayor carga atencional para poder resolverlos.

La falta de una relación significativa entre la memoria de corto plazo visoespacial y las tareas que exigen razonar a partir de mecanismos de rueda hallado en este trabajo permite afirmar que las inferencias realizadas por Hegarty y Steinhoff (1997) serían correctas y los resultados de su estudio se explicarían considerando que los sujetos con alta capacidad espacial no se servían del facilitador que implicaban las notas al margen de las láminas para resolver la tarea dado que eran capaces de mantener en línea y manipular la información de forma simultánea.

Globalmente, estos hallazgos corroboran la propuesta que las memorias de corto plazo y de trabajo verbales no inciden en la resolución de tareas de razonamiento mecánico de ningún tipo, tal como proponen Hegarty (2004), Hegarty y Kozhevnikov (1999), Hegarty y Sims (1994) y Hegarty y Steinhoff (1997), aunque sí puedan observarse asociaciones, sobre todo con el componente general de razonamiento mecánico y con la capacidad de razonar a partir de mecanismos de engranaje. De igual forma, la memoria de corto plazo visoespacial mostró no tener efecto sobre estos procesos de razonamiento, aunque si bien se encontraron asociaciones con la habilidad general de razonamiento mecánico y con la habilidad para resolver mecanismos de engranaje. En este sentido, sería interesante investigar cuál es el efecto de interferencias de almacenamiento de información visual o espacial sobre estas habilidades para poder tener seguridad que al razonar a partir de mecanismos no se requieran de capacidades exclusivamente de almacenamiento de información visual y espacial.

Por otro lado, los análisis confirman que las variables predictivas juegan roles distintos en cada tipo de razonamiento mecánico, como pasa con la memoria de trabajo visoespacial y la velocidad de procesamiento. Cuando se razona a partir de mecanismos de rueda, los participantes requieren de una mayor carga de procesamiento de memoria de trabajo visoespacial, como también de una mayor capacidad atencional de velocidad para el procesamiento de estímulos. Como se ha dicho antes, este tipo de problemas exige al resolutor que se represente los mecanismos del problema de rueda de manera distinta, ya que el movimiento de todo el 
mecanismo debe ser visualizado de manera conjunta, haciendo que no sea posible representar mentalmente el problema de forma fragmentada, este hecho genera que el resolutor necesite de una mayor carga ejecutiva de la memoria de trabajo visoespacial, como también de una mayor velocidad para el procesamiento de los estímulos que se presenta para poder resolverlo. Es posible que los resultados obtenidos se vinculen a características propias de la muestra, como el hecho de que esté conformada exclusivamente por estudiantes de Psicología. Es posible que esos resultados varíen en una población con mayor nivel de especialización, como podría ser una muestra conformada por estudiantes universitarios de la carrera de Ingeniería.

Estudios posteriores podrían abocarse al análisis del desarrollo de las variables implicadas incluyendo participantes de distintas edades o incluso utilizando un diseño de tipo longitudinal.

\section{Conflicto de intereses}

No existen conflictos de interés para ser sometido a evaluación por parte de la revista.

\section{Responsabilidad ética}

Los datos de los participantes son anónimos y confidenciales, y no aparecen datos más allá de la edad y el género. El estudio fue evaluado y aprobado por el comité de ética de la Facultad de Psicología de la Universidad de Buenos Aires.

\section{Agradecimientos}

La investigación fue llevada a cabo con el financiamiento del Consejo Nacional de Investigaciones Científicas y Técnicas (CONICET).

\section{Contribución de autoría}

IIR, JF, AC, GC, AAD, JPB participaron en la concepción y diseño del estudio, recolección de datos, interpretación de los datos, discusión y revisión final del manuscrito

\section{Referencias}

An, Y., Feng, L., Zhang, X., Wang, Y., Wang, Y., Tao, L., ... \& Xiao, R. (2018). Patterns of cognitive function in middle-aged and elderly Chinese adults-findings from the EMCOA study. Alzheimer's Research \& Therapy, 10(1), 93. doi: 10.1186/s13195-0180421-8

Ackerman, P. L. (2014). Nonsense, common sense, and science of expert performance: Talent and individual differences. Intelligence, 45, 6-17. doi: 10.1016/j.in tell.2013.04.009

Andersen, L. (2014). Visual-Spatial Ability: Important in STEM, Ignored in Gifted Education. Roeper Review, 36(2), 114-121. doi: 10.1080/02783193.2014.884198

Arribas, D., Santamaría, P., Sánchez-Sánchez, F., \& Fernández-Pinto, I. (2013). Batería para la evaluación de las aptitudes, BAT. Madrid: TEA Editores.

Baddeley, A. D. (2010). Working memory. Current Biology, 20(4), 136-140. doi: 10.1016/j.cub.2009. 12.014

Baddeley, A. D. (2018). Exploring Working Memory: Selected Works of Alan Baddeley. Abingdon, UK: Routledge.

Baddeley, A. D., \& Hitch, G. J. (1974). Working memory. In G. H. Bower (Ed.), The Psychology of Learning and Motivation: Advances in Research and Theory (Vol. 8, pp. 47-90). New York: Academic Press.

Barreyro, J. P., Injoque-Ricle, I., Formoso, J., \& Burin, D. (2019). Computerized Working Memory Battery (BIMeT-V): Studying the Relation between Working Memory, Verbal Reasoning and Reading Comprehension. Trends in Psychology. 27(I), 53-67. doi: 10.9788\tp2019.1-05

Bennett, G. K., Seashore, H. G., \& Wesman, A. G. (1992). Tests de Aptitudes Diferenciales. Buenos Aires: Paidós.

Conway, A. R. A., Cowan, N., Bunting, M. F., Therriault, D. J., \& Minkoff, S. R. B. (2002). A latent variable analysis of working memory capacity, short-term memory capacity, processing speed, and general fluid intelligence. Intelligence, 30(2), 163-183. doi: 10.10 16/S0160-2896(01)00096-4 
Corsi, P. M. (1972). Human memory and the medial temporal region of the brain. Montreal: McGill University Press.

Demetriou, A., Spanoudis, G., Shayer, M., Ven, S. van der, Brydges, C. R., Kroesbergen, E., ... \& Swanson, H. L. (2014). Relations between speed, working memory, and intelligence from preschool to adulthood: Structural equation modeling of 14 studies. Intelligence, 46, 107-121. doi: 10.1016/j.intell.201 4.05.013

Engelhardt, L. E., Mann, F. D., Briley, D. A., Church, J. A., Harden, K. P., \& Tucker-Drob, E. M. (2016). Strong genetic overlap between executive functions and intelligence. Journal of Experimental Psychology: General, 145(9), 1141-1159. doi: 10.1037/xge000 0195

English, L. D. (2016). STEM education K-12: perspectives on integration. International Journal of STEM Education, 3(1), 1-8. doi: 10.1186/s40594016-0036-1

Formoso, J., Jacubovich, S., Injoque-Ricle, I., \& Barreyro, J. P. (2018). Resolution of arithmetic problems, processing speed and working memory in children. Trends in Psichology, 26(3), 1249-1266. doi: 10.9788/TP2018.3-05en

Fry, A. F., \& Hale, S. (2000). Relationships among processing speed, working memory, and fluid intelligence in children. Biological psychology, 54(13), 1-34. doi: 10.1016/S0301-0511(00)00051-X

Hegarty, M. (2004). Mechanical reasoning by mental simulation. Trends in Cognitive Sciences, 8(6), 280285. doi: 10.1016/s1364-6613(04)00100-7

Hegarty, M. (2010). Components of Spatial Intelligence. In B. H. Ross (Ed.), The psychology of learning and motivation (pp. 265-297). San Diego, CA: Academic Press. doi: 10.1016/S0079-7421(10) 52007-3

Hegarty, M., \& Kozhevnikov, M. (1999). Spatial abilities, working memory and mechanical reasoning. In J. S. Gero \& B. Tversky (Eds.), Visual and spatial reasoning in design (pp. 223-257). Sydney, AU: Key Center for Design Computing and Cognition, University of Sydney.

Hegarty, M., \& Sims, V. K. (1994). Individual differences in mental animation during mechanical reasoning.
Memory \& Cognition, 22(4), 411-430. doi: 10.3758/ bf03200867

Hegarty, M., \& Steinhoff, K. (1997). Individual differences in use of diagrams as external memory in mechanical reasoning. Learning and Individual Differences, 9(1), 19-42. doi: 10.1016/s1041-6080(97)90018-2

Hegarty, M., \& Tarampi, M. R. (2015). Teaching Spatial Thinking: Perspectives from Cognitive Psychology. Teaching Spatial Thinking from Interdisciplinary Perspectives. New México. Recuperado de http:// ceur-ws.org/Vol-1557/paper8.pdf

Injoque-Ricle, I., Barreyro, J. P., Formoso, J., \& Burin, D. (2018). Working memory, processing speed and general intelligence: Possible models of relations with visuospatial working memory using the visuospatial Computerized Working Memory Battery (BIMeT-VE). Trends in Psychology, 26(1), 413-427. doi: 10.9788/tp2018.1-16

Jensen, A. R. (2006). Clocking the mind: Mental chronometer individual differences. Oxford, UK: Elsevier.

Kelley, T. R., \& Knowles, J. G. (2016). A conceptual framework for integrated STEM education. International Journal of STEM Education, 3(1), 11. doi: 10.1186/s40594-016-0046-z

Liu, A. S., \& Schunn, C. D. (2017). Applying math onto mechanisms: Mechanistic knowledge is associated with the use of formal mathematical strategies. Cognitive Research: Principles and Implications, 2(1), 6. doi: 10.1186/s41235-016-0044-1

Mella, N., Fagot, D., Lecerf, T., \& Ribaupierre, A. de (2015). Working memory and intraindividual variability in processing speed: A lifespan developmental and individual-differences study. Memory \& cognition, 43(3), 340-356. doi: 10.3758/s13421-014-0491-1

Rouse, W. B., \& Morris, N. M. (1986). On looking into the black box: Prospects and limits in the search for mental models. Psychological bulletin, 100(3), 349363. doi: 10.1037/0033-2909.100.3.349

Sheppard, L. D., \& Vernon, P. A. (2008). Intelligence and speed of information-processing: A review of 50 years of research. Personality and Individual Differences, 44(3), 535-551. doi: 10.1016/j.paid. 2007.09.015 
Stieff, M., \& Uttal, D. H. (2015). How Much Can Spatial Training Improve STEM Achievement? Educational Psychology Review, 27(4), 607-615. doi: 10.1007/ s10648-015-9304-8

Wai, J., \& Kell, H. J. (2017). What Innovations Have We Already Lost?: The Importance of Identifying and Developing Spatial Talent. In M. Khine (Eds), Visualspatial Ability in STEM Education (pp. 109-124). Springer, Cham. doi: 10.1007/978-3-319-44385-0_6
Wechsler, D. (2003). WAIS III: Test de Inteligencia para Adultos. Buenos Aires: Paidós.

Williams, M. D., Hollan, J. D., \& Stevens, A. L. (1983). Human reasoning about a simple physical system. Mental models. In D. Gentner \& A. L. Stevens (Eds), Mental models (pp. 131-154). New York: Psychology Press.

\section{Irene Injoque-Ricle}

Departamento de Procesos Básicos, Instituto de Investigaciones, Facultad de Psicología, Universidad de Buenos Aires (UBA), Consejo Nacional de Investigaciones Científicas y Técnicas (CONICET), Argentina

Investigadora del CONICET. Docente de la Universidad de Buenos Aires.

ORCID: 0000-0002-7043-677X

Autor corresponsal: iinjoque@psi.uba.ar

\section{Jésica Formoso}

Departamento de Procesos Básicos, Instituto de Investigaciones, Facultad de Psicología, Universidad de Buenos Aires (UBA), Consejo Nacional de Investigaciones Científicas y Técnicas (CONICET), Argentina

Becaria Posdoctoral del CONICET. Docente de la Universidad de Buenos Aires.

ORCID: 0000-0003-3062-4036

jformoso@psi.uba.ar

\section{Alejandra Calero}

Departamento de Procesos Básicos, Instituto de Investigaciones, Facultad de Psicología, Universidad de Buenos Aires (UBA), Consejo Nacional de Investigaciones Científicas y Técnicas (CONICET), Argentina

Becaria Posdoctoral del CONICET. Docente de la Universidad de Buenos Aires.

ORCID: 0000-0001-7197-1320

acalero@psi.uba.ar

\section{Guido Caruso}

Departamento de Procesos Básicos, Instituto de Investigaciones, Facultad de Psicología, Universidad de Buenos Aires (UBA), Argentina Estudiante de Psicología de la Universidad de Buenos Aires, Argentina.

ORCID: 0000-0002-8591-6215

guido_181193@outlook.com

Andrea Álvarez Drexler

Departamento de Procesos Básicos, Instituto de Investigaciones, Facultad de Psicología, Universidad de Buenos Aires (UBA), Argentina Docente de la Universidad de Buenos Aires, Argentina.

aldrexa@yahoo.com.ar

\section{Juan Pablo Barreyro}

Departamento de Procesos Básicos, Instituto de Investigaciones, Facultad de Psicología, Universidad de Buenos Aires (UBA), Consejo Nacional de Investigaciones Científicas y Técnicas (CONICET), Argentina

Investigador del CONICET. Docente de la Universidad de Buenos Aires, Argentina.

ORCID: 0000-0002-1606-1049

jbarreyro@psi.uba.ar 